\title{
Primary Transitional Cell Carcinoma of the Renal Pelvis in a Dog
}

\author{
Ju-yong Park ${ }^{* * * *}$ and Min-Hee Kang ${ }^{* 1}$ \\ *Department of Veterinary Internal Medicine, College of Veterinary Medicine, Konkuk University, \\ 120 Neungdong-ro, Gwangjin-gu, Seoul 05029, Korea \\ **Shine Animal Medical Center, 450 Songpa-daero, Songpa-gu, Seoul 05625, Korea
}

(Received: May 26, 2020 / Revised: July 16, 2020 / Accepted: August 03, 2020)

\begin{abstract}
A 12-year-old dog was evaluated for depression and anorexia. The results of complete blood count and serum biochemistry were normal. Survey radiographs revealed markedly enlarged right kidney and abdominal ultrasound showed a renomegaly and hydronephrosis filled with mixed echogenic fluid. Primary renal tumor was suspected in this dog. Nephroureterectomy of the right kidney was performed, and no regional metastases have been identified. Transitional cell carcinoma (TCC) arising from renal pelvis accompanied hydronephrosis of the right kidney was diagnosed on histology. TCC usually affects trigone of the bladder, and renal origin of TCC is a very rare finding in dogs. This is the first case report to present the clinical features, diagnostic imaging findings and histopathological characteristics of a dog with TCC originating from the renal pelvis in Korea.
\end{abstract}

Key words : dog, kidney neoplasm, renal pelvis, transitional cell carcinoma.

\section{Introduction}

Primary renal tumors are less frequent and are reported as less than $2 \%$ of all dog cancers (15). Among primary renal tumors, renal cell carcinoma is the most frequently diagnosed malignant renal epithelial tumor, and other tumors such as transitional cell carcinoma (TCC), nephroblastoma, lymphoma and sarcomas are relatively rare in dogs (11).

It is well known that TCC is the most common type of bladder tumor with papillary formations and invasiveness (15). TCC can originate in the renal pelvis and rarely occurs in dogs, cats and humans $(6,8,14)$. Painless, apparent hematuria is the most common clinical sign in human renal pelvis TCC (7). Hematuria may occur in dogs and cats, but other non-specific clinical signs are reported more often, including depression, loss of appetite, weight loss, and abdominal pain $(4,8,9)$. Renomegaly or abdominal mass is commonly found, and diagnosis requires radiographs, abdominal ultrasonography or computerized tomography scan or magnetic resonance imaging, and histopathological confirmation $(14,15)$. At the time of diagnosis, metastasis to the lungs is common, so the prognosis for this primary renal tumor is poor (15). Complete surgical resection is an ideal treatment option in the absence of metastases, and adjunctive systemic therapy is required in the cases of metastatic tumors $(9,11)$.

This report describes a renal pelvis TCC that occurs rarely in dogs. The clinical and diagnostic features and long-term follow-up of renal pelvis TCC in a dog after the nephroureterectomy were described for the first time in Korea.

${ }^{1}$ Corresponding author.

E-mail : maho79@naver.com

\section{Case}

A 12-year-old, $5 \mathrm{~kg}$, spayed female mixed-breed dog was evaluated for depression and anorexia for 4 weeks. On presentation, the dog was alert and responsive with body condition score of $4 / 9$. Physical examination did not reveal any abnormalities. Laboratory tests such as a complete blood count (CBC), serum biochemical examination, and thorax/ abdominal radiographs were performed initially. Results of CBC were within reference limits. Mildly elevated alkaline phosphate (263 U/L; reference interval, 47-254 U/L) was observed. Survey abdominal radiographs revealed markedly enlarged soft tissue opacity right kidney with abdominal mass effect, suggestive of hydronephrosis or a renal mass. The right kidney was measured by $7 \mathrm{~cm} \times 7.5 \mathrm{~cm} \times 6.5 \mathrm{~cm}$ (approximately 4.5 times the length of L2), which compressed the abdominal organs to the left (Fig 1A). Other radiographic findings were within normal limits. Ultrasonography was performed to further evaluate the abdomen. Renal ultrasound revealed marked increase in size and cavitation of the right kidney containing mixed echogenic materials and hydroureter. The normal kidney structure such as cortex, medullar, and pelvis were completely lost, and a lot of fluid had accumulated in the kidney (Fig 1B). In doppler ultrasound, no blood flow was detected in any part of the kidney. No abnormalities were detected in the bladder. Urine specific gravity, dipstick tests, and results of the other urinalysis were unremarkable. An ultrasound-guided fine needle aspiration biopsy (FNAB) of the deformative right kidney was performed, but only non-diagnostic samples such as necrotic debris were obtained. Differential diagnoses at this time included ureteral obstruction of unknown etiology and primary renal neoplasms such as renal cell carcinoma.

The owners elected an exploratory laparotomy for possi- 


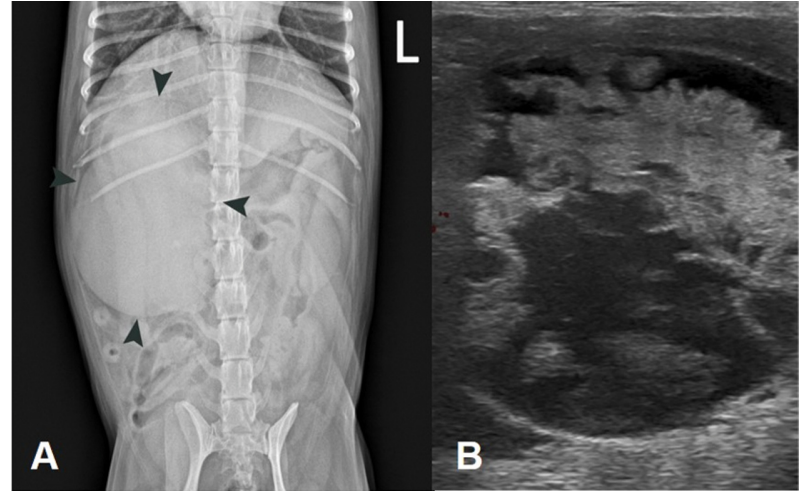

Fig 1. Ventrodorsal abdominal radiograph (A) showing the enlarged right kidney (black arrowheads). Doppler ultrasonography (B) showing absence of blood flow and fluid accumulation in the cavitated right kidney.

ble resection of the deformed kidney. The dog was premedicated with alfaxalone $(2 \mathrm{mg} / \mathrm{kg} \mathrm{BW}$, IV, Jurox, Australia), butorphanol (0.2 mg/kg BW, IV, Myungmoon, Korea), and midazolam $(0.2 \mathrm{mg} / \mathrm{kg} \mathrm{BW}$, IV, Myungmoon, Korea), and general anesthesia was maintained by isoflurane (Hana, Korea) with $100 \%$ oxygen. Exploratory laparotomy revealed a markedly enlarged right kidney at the same time as the dilation of the right ureter. Thus, right nephroureterectomy was performed in this dog. No signs of regional metastasis were observed, and other organs, including the left kidney and bladder, appeared normal. The dog recovered well from the operation and was discharged 3 days later.

On gross examination, the right kidney inflated like a balloon and contained approximately $50 \mathrm{ml}$ of brownish serosanguineous fluid (Fig 2A). Inside layer of the kidney was filled with reddish-brown tissues with spongy texture, which very suggestive of necrosis. It was impossible to recognize the kidney structure macroscopically (Fig 2B). Representative samples of resected kidney were collected for histological examination.

Investigation of multiple sections from the kidney revealed marked locally extensive ectasia of the renal pelvis, which is closely related to the mass that developed in the renal pelvic epithelium (Fig 3). The cells displayed mild anisocytosis and anisokaryosis with moderately distinct cell borders. The cell cytoplasm was moderately eosinophilic. A moderate number of atypical mitotic forms were found. No evidence of blood vessel or lymphatic system invasion was detected. In areas of marked ectasia of the renal pelvis, there was moderate to severe atrophy of the overlying renal cortex and medulla,

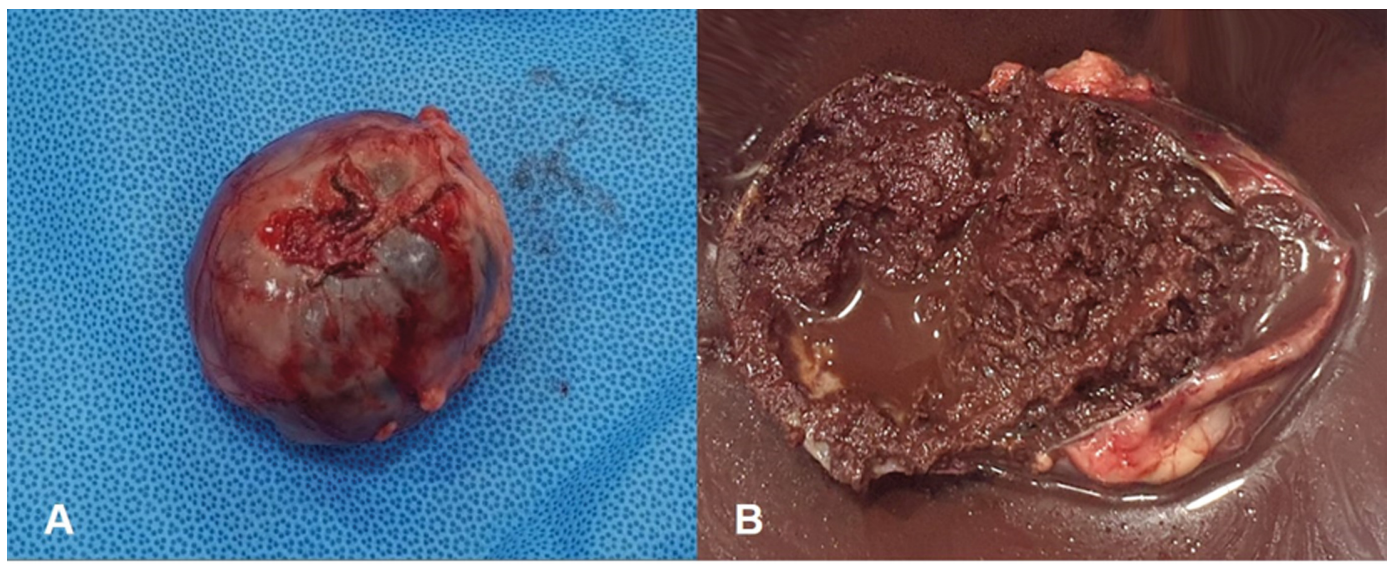

Fig 2. Gross features of the right kidney at the time of surgery in a dog with renal pelvic TCC. (A) Enlarged right kidney, inflated balloon-like shape, was measured $7 \mathrm{~cm} \times 7.5 \mathrm{~cm} \times 6.5 \mathrm{~cm}$. A serosanguineous fluid was identified (B) upon resection of the cross section of the right kidney. The remaining brownish tissues had a spongy texture, suggesting necrosis. Normal renal architectures were completely lost.

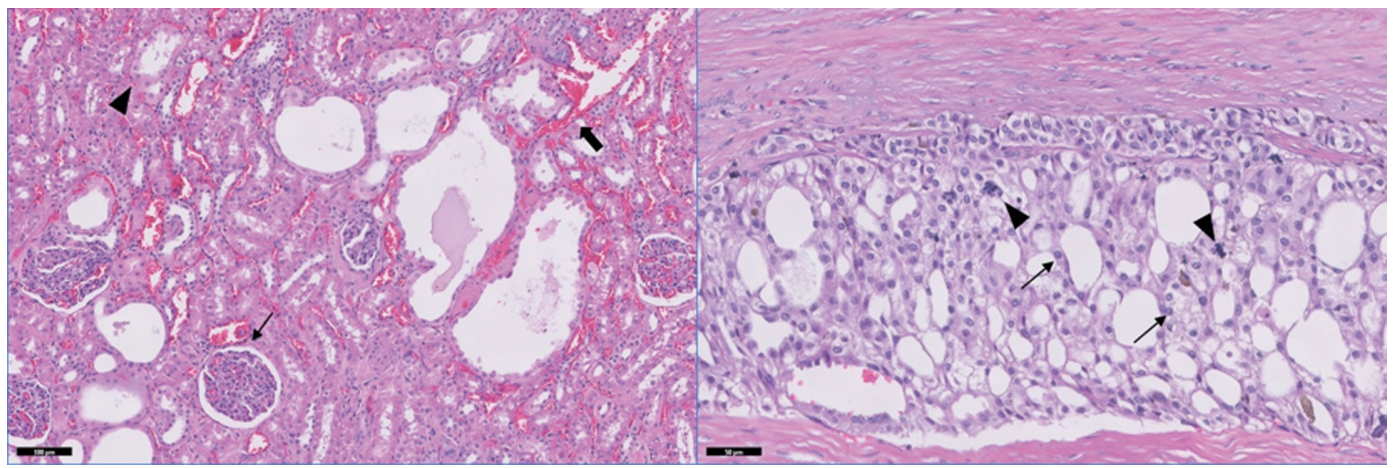

Fig 3. Histopathology of a renal pelvic TCC in a dog. (A) Note the massive hemorrhage (thick arrow), ectasia of the proximal convoluted tubule (arrowhead), and thickening of the Bowman's capsule (thin arrow). (B) Note the tumor cells (arrows) and mitotic figures (arrowheads) in the transitional epithelium. Hematoxylin and eosin stain, Bar $=100 \mu \mathrm{m}(\mathrm{A})$ and $50 \mu \mathrm{m}(\mathrm{B})$. 
moderate interstitial infiltrate of myeloid precursors, and fewer lymphocytes and plasma cells. In the region where the renal cortex and medulla was less effected, multifocal areas of mild to moderate ectasia of proximal convoluted tubules were observed, which was likely secondary to mass and hydronephrosis. Some glomeruli revealed thickening of Bowman's capsule. Locally extensive hydronephrosis along with adjacent renal cortical and medullary atrophy was observed. Histologic evaluation was consistent with transitional cell carcinoma (TCC) arising from the renal pelvis, with necrosis, hemorrhage, and fibrous encapsulation.

One month after the surgery, clinical signs of the dog such as depression and anorexia improved completely. No further treatment was implemented. Transient detection of occult blood and leukocytes in the urine was detected 1 month after surgery. Follow-up radiographs, blood works, and urinalyses were performed at 1, 3 and 12 months after the surgery and the results remained within normal ranges. In 12 months follow up, no recurrence was seen and the dog was in a healthy clinical condition.

\section{Discussion}

Diagnosis of renal pelvis TCC is challenging due to nonspecific clinical signs. According to the previous reports $(1,8)$, hematuria considered the most common problem on admission of the primary renal tumors, but only about a third of dogs were observed. In this case, the dog showed chronic anorexia and depression without any urologic signs such as hematuria and proteinuria.

Laboratory examinations such as $\mathrm{CBC}$ and serum chemistry findings were non-remarkable $(1,15)$ and urinalysis including urine sediment is also considered unreliable because shedding of the renal epithelium is not consistent $(4,9)$. Previous report suggested a combination of ultrasonography and imaging-guided FNAB as a useful diagnostic tool (9). However, in this case, FNAB was unable to provide relevant cell samples due to necrosis of the kidney, massive cavitation and severe hydronephrosis.

Mostly reported cases of renal pelvis TCC showed solid mass lesions in the renal pelvis and infiltration into the renal tissues $(5,9,11)$. As in this dog, renal pelvis TCC can also cause hydronephrosis $(9,16)$, which has also reported in cats $(12,14)$. Renal pelvis dilation, so called 'hydronephrosis' on ultrasound, has been associated with obstruction of outflow, neoplasia, ectopic ureter, or pyelonephritis $(2,13)$. In this dog, the differentials include hydronephrosis secondary to urethral obstruction of unknown etiology and primary renal neoplasm.

Primary renal tumors have various histological types and more than fifty percent of malignant renal tumors are epithelial-derived tumors. Two typical types of epithelial-derived malignant renal tumors are renal cell carcinoma and renal TCC. Pathologically, renal TCC are derived from transitional cells lining the renal pelvis epithelium, while renal cell carcinoma derived from proximal convoluted tubular epithelium which can be subdivided into tubular, solid, acinar or papillary types $(10,15)$.

Pulmonary metastasis of the primary renal tumor is reported in $16 \%$ to $48 \% \operatorname{dog}(1,8)$. In case of renal pelvic tumors, the incidence of metastasis has been rarely reported $(4,9)$. One study reported metastasis from the kidney capsule to the intestinal serosa and wall (3). Other study reported local metastasis of renal pelvis TCC to the surrounding omentum, bladder and intestine (11). Thus, metastases should be carefully evaluated before deciding on treatment options.

The management of renal pelvis TCC through surgical resection is most favorable in dogs without metastases. In the case of bilateral kidney involvement or metastasis, multimodality therapy was required $(1,11)$. However, it is difficult to demonstrate the effectiveness of multimodality therapy because of the relatively small number of cases. The survival time of the renal pelvis TCC varied from 3 months to 25 months in some cases $(8,9,11)$, and the beneficial effects of the chemotherapy on survival were still unclear (1). This dog was favorable prognosis after the nephroureterectomy of right kidney and the clinical condition was good after 1 year of surgery.

\section{Conclusions}

This case describes a primary renal pelvis TCC with hydronephrosis, successfully controlled with nephroureterectomy. TCC normally affect trigone of the bladder and TCC of renal origin represents a very rare finding in dogs. To our knowledge, this is the first report of a primary renal pelvis $\mathrm{TCC}$ in the dog in Korea.

\section{Acknowledgements}

This research was supported by the Bio \& Medical Technology Development Program of the National Research Foundation (NRF) funded by the Korean government (MSIT) (2016M3A9B6903437).

\section{References}

1. Bryan JN, Henry CJ, Turnquist SE, Tyler JW, Liptak JM, Rizzo SA, Sfiligoi G, Steinberg SJ, Smith AN, Jackson T. Primary renal neoplasia of dogs. J Vet Intern Med 2006; 20: 1155-1160.

2. D’Anjou MA, Bédard A, Dunn ME. Clinical significance of renal pelvic dilatation on ultrasound in dogs and cats. Vet Radiol Ultrasound 2011; 52: 88-94.

3. Dagli ML, Calderaro FF, Silva MT, Guerra JL. Squamous cell carcinoma of the renal pelvis with metastasis in a dog. $\mathrm{J}$ Comp Pathol 1997; 116: 397-402.

4. Goldsmid SE, Bellenger CR, Watson ADJ, France MP, Allan GS. Renal transitional cell carcinoma in a dog. J Am Anim Hosp Assoc 1992; 28: 241-244.

5. Grillo TP, Brandão CV, Mamprim MJ, de Jesus CM, Santos TC, Minto BW. Hypertrophic osteopathy associated with renal pelvis transitional cell carcinoma in a dog. Can Vet J 2007; 48: 745-747.

6. Hall MC, Womack S, Sagalowsky AI, Carmody T, Erickstad MD, Roehrborn CG. Prognostic factors, recurrence, and survival in transitional cell carcinoma of the upper urinary tract: a 30year experience in 252 patients. Urology 1998; 52: 594-601.

7. Kirkali Z, Tuzel E. Transitional cell carcinoma of the ureter and renal pelvis. Crit Rev Oncol Hematol 2003; 47: 155-169.

8. Klein MK, Cockerell GL, Harris CK, Withrow SJ, Lulich JP, 
Ogilvie GK, Norris AM, Harvey HJ, Richardson RF, Fowler JD, Tomlinson J, Henderson RA. Canine primary renal neoplasms: a retrospective review of 54 cases. J Am Anim Hosp Assoc 1988; 24: 443-452.

9. Militerno G, Bazzo R, Bevilacqua D, Bettini G, Marcato PS. Transitional cell carcinoma of the renal pelvis in two dogs. J Vet Med A Physiol Pathol Clin Med 2003; 50: 457-459.

10. Morris J, Dobson J. Urinary tract. In: Small animal oncology. Oxford, London: Wiley-Blackwell. 2001: 154-165.

11. Ocarino NM, Souza PC, Boeloni JN, Lavalle GE, Souza EM, Pereira LC, Serakides R. Transitional cell carcinoma of the renal pelvis in a dog with combined treatment with unilateral nephrectomy and carboplatin. Braz J Vet Pathol 2009; 2: 8084.
12. Raffan E, Kipar A, Barber PJ, Freeman AI. Transitional cell carcinoma forming a perirenal cyst in a cat. J Small Anim Pract 2008; 49: 144-147.

13. Slatter DH. Kidneys. In: Textbook of small animal surgery, 3rd ed. Philadelphia: WB Saunders Co. 2003: 1606-1619.

14. Wimberley HC, Lewis RM. Transitional cell carcinoma in the domestic cat. Vet Pathol 1979; 16: 223-228.

15. Withrow SJ, Vail DM, Page RL. Tumors of the Urinary System. In: Withrow \& MacEwen's small animal clinical oncology, 5th ed. St. Louis, Missouri: Elsevier. 2013: 572-582.

16. Zotti A, Corsi F, Ratto A, Petterino C. What is your diagnosis? Transitional cell carcinoma. J Am Vet Med Assoc 2010; 237: 777-778. 